\title{
Réduire la vitesse et l'empreinte écologique des déplacements de terrain
}

Retour sur deux semaines d'itinérance à vélo entre 6 Cittàslow italiennes

\section{Emmanuel Munch}

\section{OpenEdition}

Journals

Édition électronique

URL : https://journals.openedition.org/cdg/6392

DOI : $10.4000 /$ cdg.6392

ISSN : 2107-7266

\section{Éditeur}

UMR 245 - CESSMA

\section{Référence électronique}

Emmanuel Munch, «Réduire la vitesse et l'empreinte écologique des déplacements de terrain »,

Carnets de géographes [En ligne], 14 | 2020, mis en ligne le 13 janvier 2021, consulté le 21 mai 2021.

URL : http://journals.openedition.org/cdg/6392 ; DOI : https://doi.org/10.4000/cdg.6392

Ce document a été généré automatiquement le 21 mai 2021.

\section{c) (7) $९$}

La revue Carnets de géographes est mise à disposition selon les termes de la Licence Creative Commons Attribution - Pas d'Utilisation Commerciale - Pas de Modification 4.0 International. 


\title{
Réduire la vitesse et l'empreinte écologique des déplacements de terrain
}

Retour sur deux semaines d'itinérance à vélo entre 6 Cittàslow italiennes

\author{
Emmanuel Munch
}

« Entre des hommes libres, des rapports sociaux productifs vont à l'allure d'une bicyclette, et pas plus vite. » (Illich, [1975], $2004: 391$ )

1 Les travailleurs ayant besoin de se déplacer dans le cadre de leur activité professionnelle (livreurs, artisans, chefs de chantier, commerciaux...) ont généralement besoin d'effectuer leurs déplacements le plus rapidement possible pour répondre à des exigences de productivité.

2 D'un point de vue collectif et écologique, les déplacements rapides nécessitent d'importantes consommations énergétiques (Bigo, 2020), fussent-elles électriques ou carbonées et ont, par ce biais, en moyenne, un effet négatif sur la pollution : «Plus on se déplace rapidement plus on pollue ». D'un point de vue individuel et sociologique, la vitesse ne fait pas gagner du temps, mais de l'espace aux individus. Plus on circule rapidement plus on accède aisément à un ensemble d'activités et d'opportunités dispersées dans l'espace. Ainsi, parce que les déplacements rapides permettent de réaliser un plus grand nombre d'activités au quotidien, ils sont souvent associés à un sentiment d'accélération des rythmes de vie ou de manque de temps (Wiel, 2007). L'augmentation des vitesses de déplacement nous permet d'être "hypermobiles ", et autorise par ce biais des agendas toujours plus remplis. On comprend dans ces conditions pourquoi, "plus on a la possibilité d'être transporté, plus on manque de temps » (Illich, [1975], $2004:$ 402).

3 Bien qu'elles soient assez bien documentées, les deux problématiques générées par la quête de vitesse pour la productivité sont rarement mises en dialogue. Dans le cadre d'une réponse à appel à projets de l'ADEME formulée avec l'aide de plusieurs collègues de l'Université Gustave Eifel, nous cherchons à structurer une réflexion associant ces 
problématiques écologiques et sociologiques relatives à la vitesse des déplacements. Dans le cadre de cette recherche-action, nous sommes amenés à questionner l'aspiration à la décélération des rythmes de vie (et de travail) (Descarrega, Moati, 2016) en tant que levier de la transition écologique. L'objectif central du projet est de clarifier les possibilités en matière de transition écologique que pourraient induire des politiques de mobilité durable intégrant plus explicitement l'aspiration à un remplissage moins intense des agendas quotidiens. En conséquence, l'objet d'étude est positionné à l'échelle de l'ensemble des ménages urbains et des politiques de mobilité qui pourraient les concerner.

4 Pour étudier l'aspiration à la décélération des rythmes de vie en tant que potentiel levier de transition écologique, ma démarche ${ }^{1}$ m'a d'abord conduit à enquêter sur les villes membres du réseau Cittàslow en Italie. A priori, ce réseau regroupe des collectivités ayant déjà intégré des actions en lien avec l'aspiration croissante des ménages pour une forme de ralentissement de leur expérience quotidienne. Ce mouvement est né en Italie durant les années 1990, dans la continuité du mouvement de décroissance et de localisme Slow Food. Le label s'adresse à des villes moyennes de moins de 50000 habitants qui doivent s'engager à adopter des mesures qui vont dans le sens d'un urbanisme à visage humain ${ }^{2}$ et de modes de déplacements lents et peu énergivores.

Cela étant, même si l'aspiration à la décélération des rythmes de vie se cristallise de façon relativement homogène dans les Cittàslow, il s'agit d'un désir qui ne se retrouve pas équitablement au sein de toutes les catégories de population. Il est des catégories pour qui ce n'est pas l'accélération qui pose problème, mais bien la stagnation. On pensera par exemple aux retraités ou aux chômeurs. À l'inverse, les parents de jeunes enfants, les cadres, les indépendants, les plus diplômés et de manière générale les femmes (Adam, 2004) reportent à la fois de façon subjective plus fréquemment un sentiment de manque de temps, en même temps qu'ils réalisent objectivement et en moyenne un plus grand nombre d'activités au quotidien (Davies, 2003; Schwanen, 2007).

6 Dans le contenu de leur activité, et parce qu'ils sont situés à l'intersection des catégories socioprofessionnelles sensibles aux enjeux de gestion du temps quotidien, les chercheurs amenés à réaliser des enquêtes de terrain se trouvent fortement soumis aux problématiques soulevées par la vitesse de leurs déplacements (Gane, 2006). Ils subissent des contraintes d'accélération qui s'observent au travers de déplacements de terrain rapides et potentiellement polluants. L'internationalisation des travaux et des terrains de recherche nécessite souvent de prendre l'avion pour mener à bien les enquêtes dans le temps imparti. Les recherches réalisées en France, tant qu'elles ont lieu dans des agglomérations suffisamment grandes, peuvent être réalisées grâce à l'utilisation de transports ferrés. Seulement, dès lors qu'on s'intéresse à des territoires ruraux, les chercheurs sont quasiment obligés d'utiliser des modes motorisés (voiture, moto, etc.) pour accéder aux différents terrains.

7 Face aux contraintes de productivité et de rentabilité, les chercheurs ont a priori eux aussi toutes les raisons d'aspirer à la décélération de leur rythme de recherche (Stengers, 2016) lors d'enquête de terrain. Avant même l'arrivée sur le terrain, le temps qui sépare le début de la fin du terrain est déjà rempli, quelle que soit sa durée : rempli d'objectifs, de matériaux à collecter, de phénomènes à décrypter, d'instants à saisir. «En situation, la plupart d'entre nous sommes hantés par la perte de temps. Il faut rentabiliser le plus possible la durée si difficilement dégagée, chaque minute devient 
précieuse. Même ces fameux moments d'ennui, si essentiels en recherche qualitative, sont prévus et comptabilisés » (Collignon, 2010 : 75). Se déplacer plus lentement et de façon non-polluante pourrait alors devenir une nouvelle entrée pour imposer un rythme de production plus soutenable et plus favorable à la création de connaissances solides et approfondies. Réciproquement, aspirer à un rythme de production plus favorable au travail de recherche pourrait aller dans le sens de la diminution de l'empreinte écologique des travaux scientifiques. Au total, par la concrétisation de leurs aspirations, les chercheurs, et plus généralement la communauté scientifique, pourraient eux-aussi devenir des acteurs directs de la transition écologique.

8 À partir de ces quelques remarques, se dessinent des possibilités d'articulation entre la question générale de l'aspiration à la décélération et une étude plus empirique des pratiques des chercheurs lors d'enquêtes de terrain.

Ainsi, au travers de ce carnet de terrain, je ne vais pas tant rendre compte, ici, de mes enquêtes dans les Cittàslow italiennes (Munch et al., 2020). Je propose plutôt un retour réflexif qui vise à retracer ma pratique de déplacements entre mes différents terrains de recherche dans les régions de l'Emilie-Romagne, la Toscane et l'Ombrie. Par ce récit, je souhaite expliquer comment des interrogations scientifiques et théoriques sur les pratiques de déplacements ralenties ont été mises en abyme et appliquées à mes déplacements professionnels. En effet, en utilisant le vélo pour cheminer entre les différentes villes enquêtées, je me suis appliqué à moi-même des pratiques de déplacement ralenties et non-polluantes que je questionne dans le projet de recherche.

Personnellement, cette expérience me tient à cœur. D'une part, il est clair que le fait de réaliser un projet sur l'aspiration à la décélération des rythmes de vie en tant que levier de la transition écologique n'est pas un hasard pour moi. Dans ma vie personnelle, tout se passe effectivement comme si j'étais porteur de réponses assez clairement orientées face aux questions que nous nous posons scientifiquement. D'autre part, j'aime me promener à vélo. Je fais fréquemment du cyclotourisme pendant mes congés. Cela n'est évidemment pas anodin vis-à-vis de la conduite de cette recherche.

11 En me «mettant en récit » en tant que chercheur, et en couplant des questions de recherche à des pratiques personnelles, je pense qu'il devient possible de faciliter le retour réflexif sur la position personnelle que j'occupe dans la réalisation de ce projet. Plus généralement, sur le mode de l'auto-ethnographie analytique (Anderson, 2006), l'idée est de montrer que l'étude de ses déplacements à vélo en tant que chercheur (Larsen, 2014) peut avoir un intérêt dans la phase exploratoire d'une recherche sur les temporalités des mobilités. Ensuite, l'objectif est d'informer la communauté scientifique sur les avantages et les inconvénients de l'usage d'un mode écologique et lent, le vélo, pour cheminer entre des terrains d'enquêtes proches. Enfin, l'ambition est que ce retour d'expérience puisse alimenter des débats sur la portée éthique et écologique des déplacements professionnels des chercheurs (Nevins, 2014).

\section{L'utilisation réflexive d'un mode de transport lent et non-polluant pour réaliser des enquêtes de terrain}

Durant l'entre-deux-guerres et jusqu'au début des années 1950, les géographes (Paul Claval, Thomas Walter Freeman, Torsten Hägerstrand...) utilisent volontiers le vélo pour sillonner leurs terrains d'enquête (Buttimer, 1983). À l'époque, cette pratique 
correspond surtout à une mode, c'est-à-dire au développement du vélo dans l'ensemble de la société. Ce n'est pas le reflet d'un positionnement épistémologique sur la pratique du terrain en géographie. Il faut attendre les années 1980 pour que les géographes s'attardent sur la manière dont le terrain est pratiqué, en tant que problème épistémologique. En réaction à une conception purement intellectuelle de la science, l'aspect concret des démarches et leurs conditions matérielles est plus nettement pris en compte. Émerge alors l'idée qu'un contact direct avec les paysages et les hommes est indispensable pour les expliquer (Baudelle et al., 2001). Indéniablement, l'éclosion de ce mouvement épistémologique résulte aussi d'une prise de conscience des inconvénients $\mathrm{du}$ «tout automobile» et du rapport de plus en plus désincarné au territoire qui en découle (Claval, 2013).

Aujourd'hui, la question de l'intérêt heuristique et méthodologique du renoncement à la voiture pour réaliser des enquêtes de terrain est toujours d'actualité. À cette question, s'ajoute également celle de l'enjeu éthique et écologique associée au mode de transport utilisé pour rejoindre ou réaliser ces différentes enquêtes de terrain.

Pour un coût écologique nul, la marche (Lee, Ingold, 2006 ; Edensor, 2000) et le vélo (Fincham, 2006) permettent d'atteindre un niveau de flexibilité et d'accessibilité similaire à celui de la voiture, dès lors qu'on a la possibilité d'accorder plus de temps aux déplacements. Le parapente, la barque (Les êtres Loire, 2020) etc. relèvent euxaussi des mêmes logiques dès lors que la topographie du «terrain » invite favorablement à leur usage.

Seulement, la plupart des chercheurs qui utilisent ces modes de déplacement lents et non polluants n'y adossent pas nécessairement un questionnement écologique. Bien souvent, ces modes de transport sont avant tout utilisés parce qu'ils sont nécessaires pour effectuer des relevés biologiques, atmosphériques, aquatiques - voire ethnographiques ou géographiques dans ce que la littérature anglo-saxonne qualifie de mobile researching ${ }^{3}$ (Büscher et al., 2010). Ils servent les intérêts de la recherche en itinérance, sans questionner les conséquences écologiques de l'itinérance. À titre d'exemple récent, on peut néanmoins mentionner l'expérience de Nicolas Plain ${ }^{4}$ qui, dans le cadre d'une recherche sur la pollution atmosphérique en milieu alpin, a sillonné en 2019 les Alpes de Cannes à Salzbourg sans la moindre émission de $\mathrm{CO}_{2}$, en utilisant le parapente, le vélo et la marche. Afin de sensibiliser sur la possibilité de faire du terrain en sciences environnementales sans émettre de polluants, sa démarche a été largement relayée dans les médias. Cependant, ici encore, cette initiative n'a été rendue possible que parce que les objectifs scientifiques étaient localisés au sein de l'itinérance même. Il n'est pas du tout évident de penser que sa sensibilisation aux enjeux écologiques de la pratique du terrain eut été possible s'il avait dû effectuer des observations, non pas à chaque instant de ses déplacements (il effectuait notamment des relevés de pollutions atmosphériques en parapente), mais à l'arrivée, disons dans une ville ou un village situé au bout de chacun de déplacements.

Dans le domaine plus circonscrit de l'étude des mobilités, l'approche autoethnographique est privilégiée (Brown, Spinney, 2010) afin d'interroger sa propre pratique du déplacement au cours de l'itinérance à faible allure, souvent à vélo ou à pied. Ces recherches plus proches de l'anthropologie servent la plupart du temps à analyser et comprendre le rapport sensible qui se développe entre le corps, l'espace et le rythme (Bissell, 2010) à mesure que l'on se déplace à la force des jambes. 
17 En termes de focalisation sur leur objet d'étude, lorsque les chercheurs étudient la lenteur de leurs déplacements, ils ne positionnent jamais le curseur à l'échelle de leur vie quotidienne et de leur vie professionnelle de chercheurs en action. Les déplacements étudiés ont une fonction expérimentale. Ils sont hors-sol ; comme si la lenteur des déplacements ne pouvait être justifiée et donc étudiée qu'en s'extrayant des contraintes de la vie quotidienne et professionnelle, qu'en l'absence d'activités situées au bout du déplacement qui nécessitent de se hâter. Deuxièmement, les chercheurs qui analysent de façon réflexive leurs pratiques de déplacement ne traitent quasiment jamais de la portée écologique de ces derniers, mais plutôt de leurs performances méthodologique ou heuristique $\mathrm{du}$ point de vue du rapport sensible auquel ils permettent d'accéder. La portée heuristique de leur «déplacement ralenti » n'est jamais positionnée face à la demande sociale pour la décélération des rythmes de vie.

18 En conséquence, le présent article a vocation à aller au-delà des postures méthodologiques usuelles sur l'itinérance, en déplaçant l'oculaire des préoccupations scientifiques et épistémologiques. Dans le cadre de mon expérience en Italie, je rends compte de la façon dont il est possible d'utiliser le vélo, non pas pour effectuer des « relevés », mais bien pour se rendre lentement et écologiquement sur un ou plusieurs lieux d'enquête. En élargissant la réflexion à l'ensemble des déplacements de terrain, je propose d'ouvrir des perspectives plus étendues sur la portée heuristique et écologique des déplacements professionnels des chercheurs. En effet, les résultats issus de ce travail pourraient potentiellement s'appliquer à nombre d'enquêtes de terrain puisque, par définition, elles prennent toutes place en dehors du bureau du chercheur et nécessitent par suite un déplacement qui pourrait être effectué, pour tout ou partie, à vélo.

\section{L'itinérance à vélo en Italie entre 6 Cittàslow}

\section{Descriptif, avantages et inconvénients de l'itinérance à vélo par rapport à l'automobile}

19 Concrètement, pour adosser mes questions de recherche à ma pratique réflexive du terrain, entre le 22 octobre et le 5 novembre 2019, je me suis déplacé à vélo entre les différentes Cittàslow que j'ai enquêtées en Italie ${ }^{5}$. Le 22 octobre, je suis parti à vélo de Bologne avec le matériel nécessaire dans mes sacoches pour réaliser des enquêtes dans six villes d'Emilie-Romagne, de Toscane et d'Ombrie, sur le mode de l'itinérance (figure 1). 
Figure 1. Itinéraire d'enquête entre 6 Cittàslow italiennes

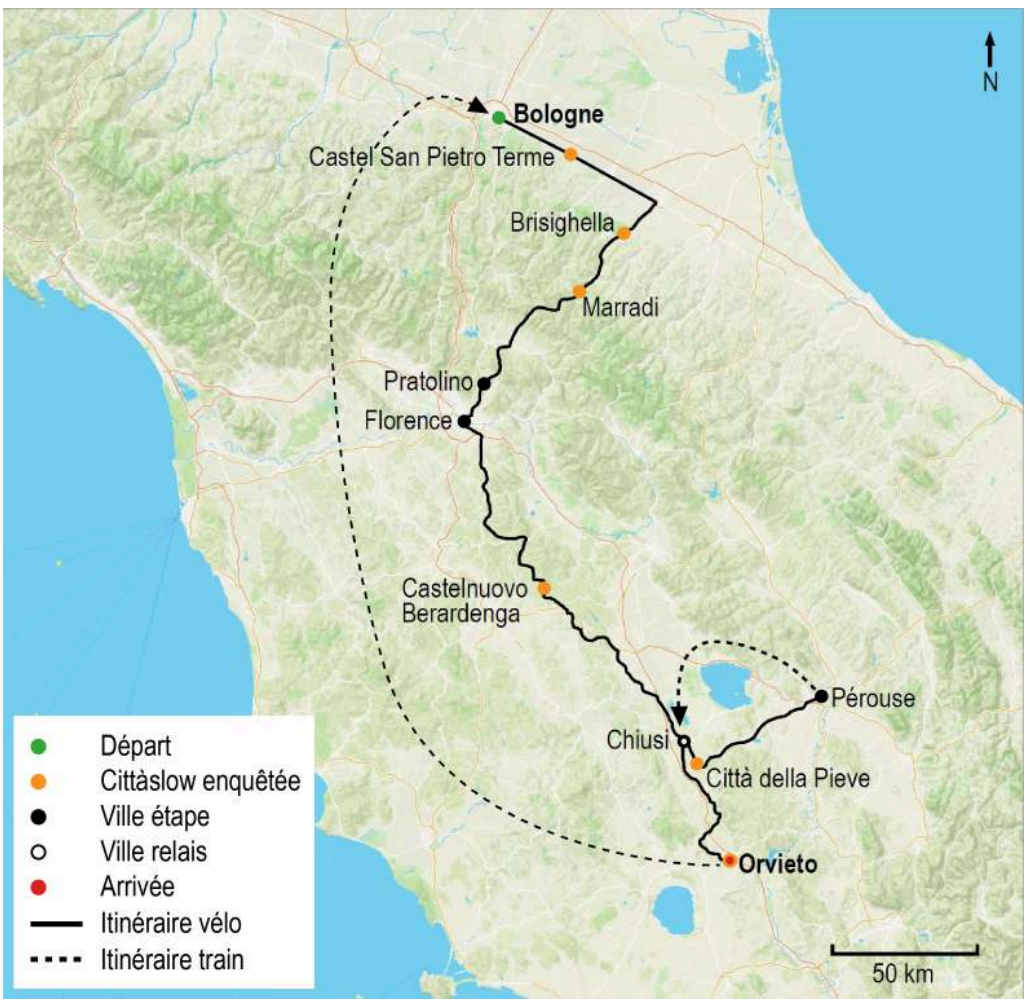

Réalisation : Maxime Sauder et Emmanuel Munch. Source : Carte mondiale OpenStreetMap. (2020). Lecture : Chaque pastille colorée sur la carte correspond à une halte d'au moins une nuit. Chiusi est une ville relais. Je n'ai pas séjourné dans cette ville. Je n'y ai fait qu'un passage au retour de Pérouse, afin de reprendre mon itinéraire cyclable en direction d'Orvieto.

Durant deux semaines, j'ai parcouru $427 \mathrm{~km}$ avec mon vélo entre Bologne (mon lieu de résidence en Emilie-Romagne) et Orvieto (le siège social de l'association Cittàslow en Ombrie). J'ai transporté tout le matériel nécessaire (ordinateur, enregistreur, appareil photo, cartes, vêtements...) dans mes sacoches (figure 2). 
Figure 2. Mon vélo Bottechia (Bologne, 22 octobre 2019)

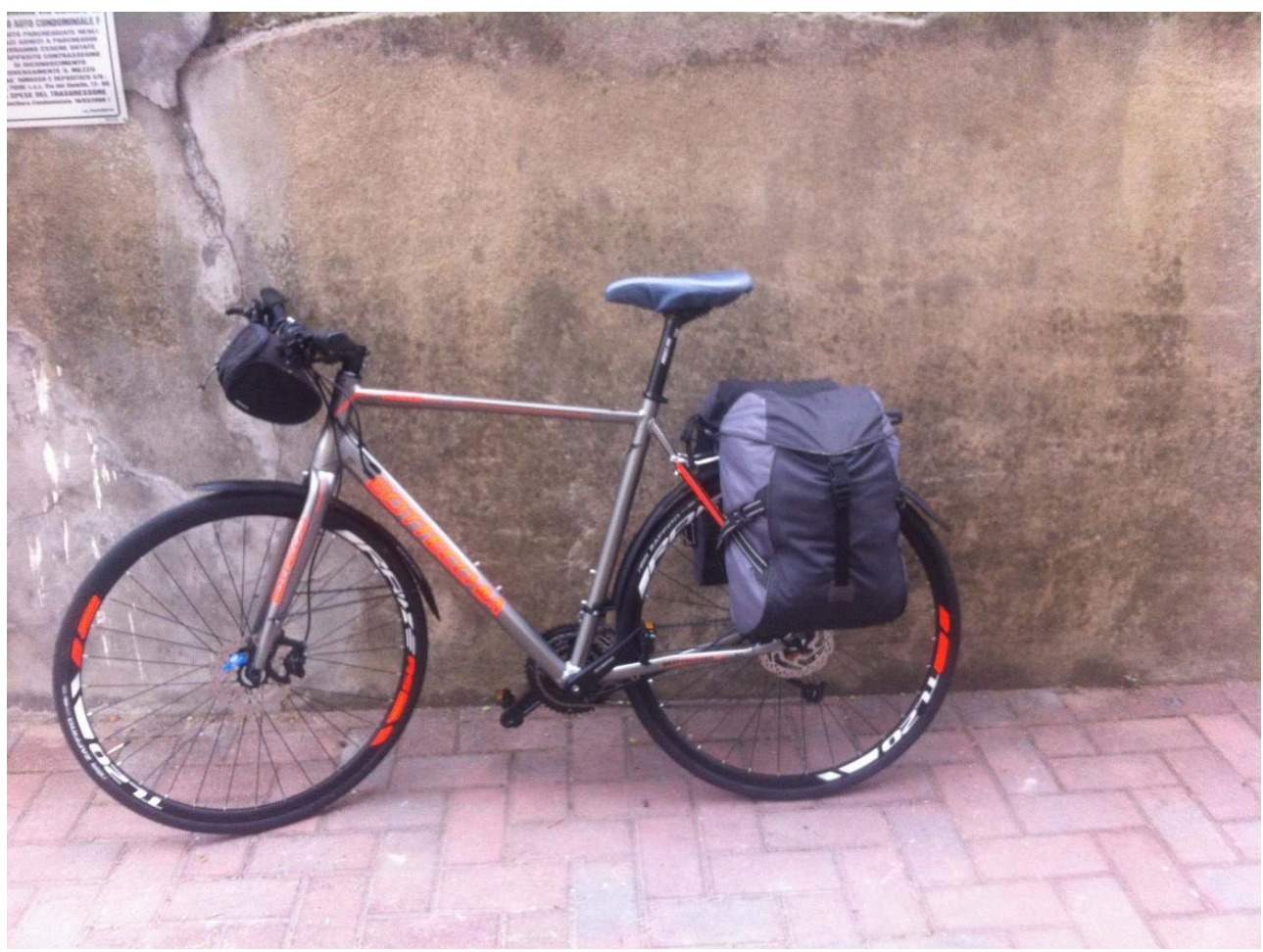

Crédits : Emmanuel Munch.

21 La plupart du temps, je changeais de villes tous les jours (hors jours fériés et week-end). Selon les objectifs à atteindre, je réalisais quotidiennement entre 20 et $74 \mathrm{~km}$ de vélo par jour, passais entre 1 et 6 heures par jour sur ma bicyclette (Tableau 1), faisais dans certains cas plus de 2000 mètres de dénivelé positif dans la même journée ou d'autres jours - comme pour rejoindre Florence - me laissais simplement descendre en " roue libre » (Figure 3). Aucun jour, aucun de mes déplacements ne ressemblait vraiment à un autre. Les paysages traversés étaient aussi variés qu'éprouvants, mes douleurs à la paume des mains aussi oscillantes que mes humeurs.

Figure 3. Profil altimétrique du voyage

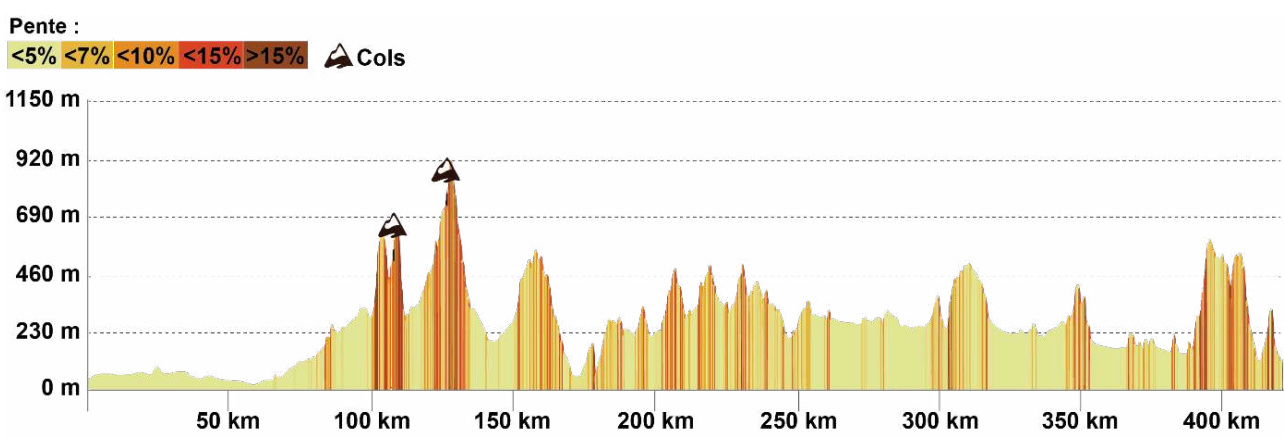

Réalisation : Maxime Sauder et Emmanuel Munch. Source : réalisé à partir du site internet openrunner.com

Cette description contraste certainement avec la monotonie, la fiabilité et la sécurité des déplacements que j'aurais pu comparativement ressentir en voiture. À vélo, j'ai circulé fréquemment sur des routes dangereuses ${ }^{6}$ à fort trafic (figure 4). 
Figure 4. Circulation sur la bande d'arrêt d'urgence d'une route nationale (en direction de Pérouse, 30 octobre 2019)

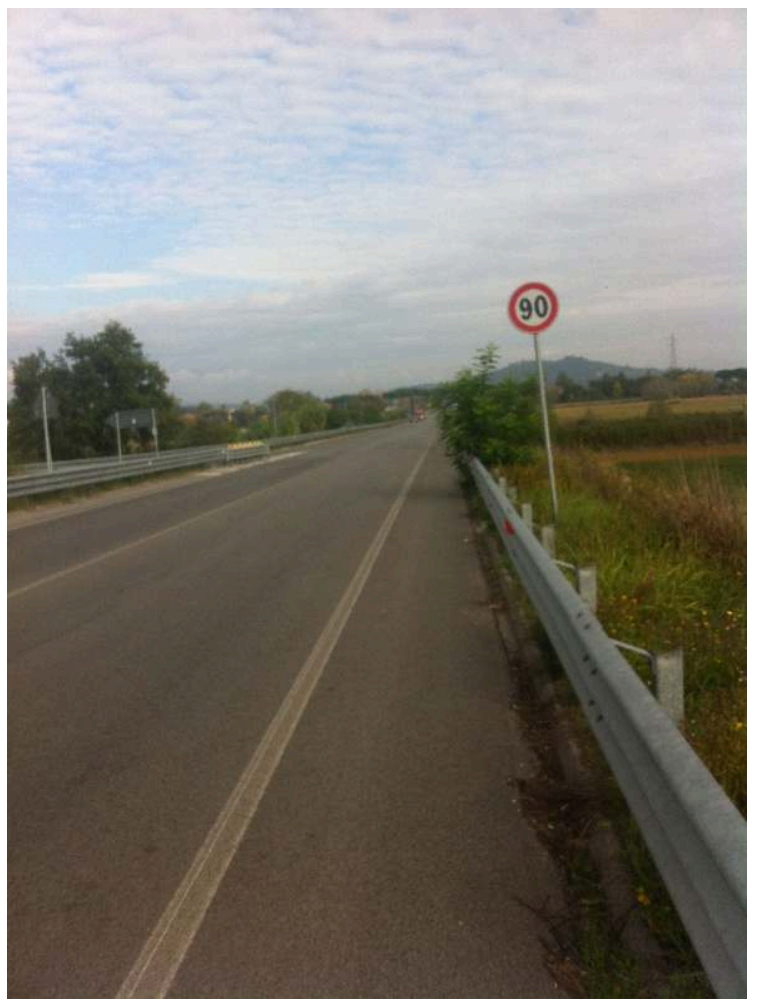

Crédits : Emmanuel Munch.

23 De l'autre côté de la balance, face à la difficulté liée à la pratique du vélo sur des voies de circulation dédiées à l'usage de l'automobile, on peut en revanche mentionner la facilité de stationnement du vélo par rapport à la voiture (figure 5). 
Figure 5. Stationnement sur le parvis de la cathédrale d'Orvieto (Orvieto, 30 octobre 2019)

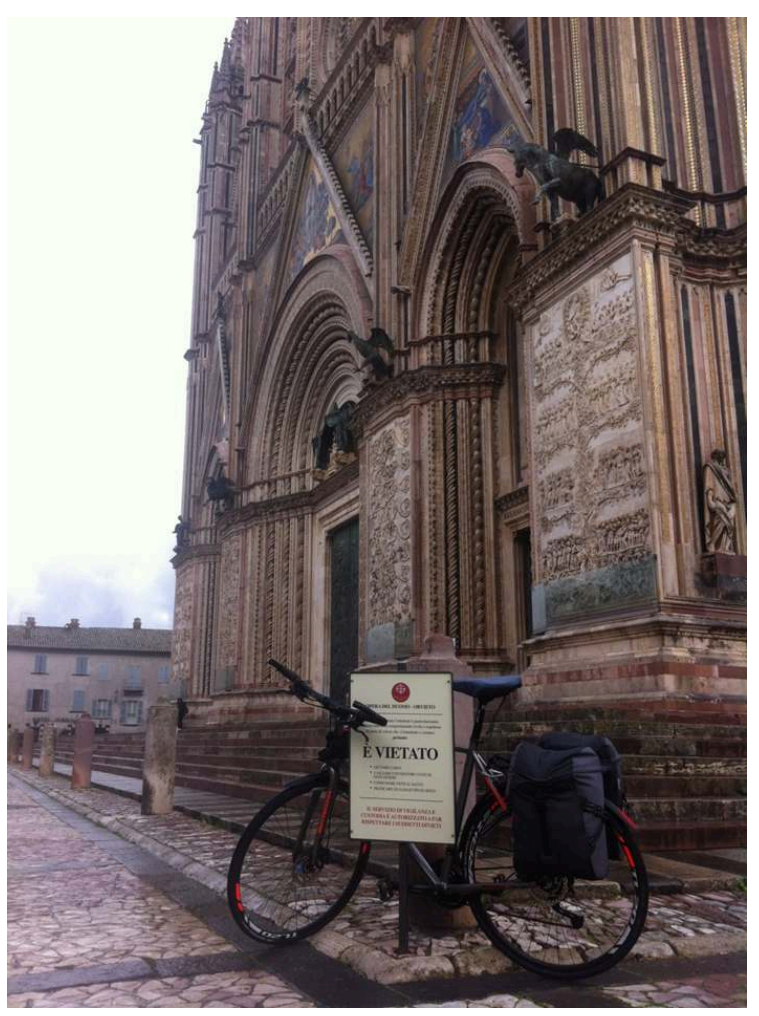

Crédits : Emmanuel Munch.

En mettant de côté les avantages liés à la facilité de stationnement du vélo et les inconvénients liés à la relative insécurité de cette pratique sur certaines routes (tous deux assez difficilement chiffrables), il devient possible de tenter une rapide comparaison économique des avantages et inconvénients de mon voyage à vélo par rapport à un voyage équivalent (en destination et distance) en voiture.

Tableau 1. Descriptif de l'itinérance (Aller) à vélo et comparaison des "coûts" du voyage par rapport à un voyage fictif en voiture

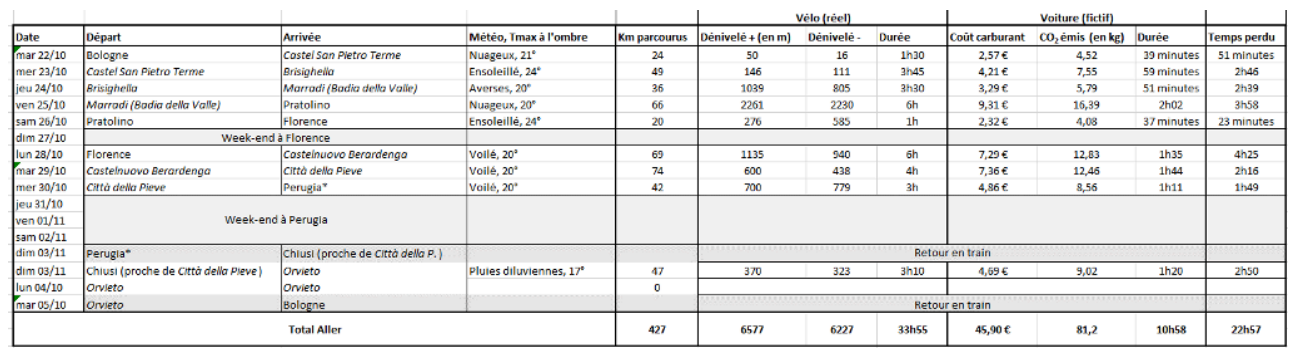

Lecture :

Graphie italique : Cittàsslow enquêtées ; Graphie romaine : Villes étape ou ville relais ; * Entretien exploratoire avec Paola de Salvo à l'Université de Pérouse.

Durée vélo : temps total du point de départ au point d'arrivée, pause(s) comprise(s) ; Durée voiture : hors congestion en zone urbaine et temps pour stationner.

Coût fictif du déplacement en voiture calculé à partir de viamichelin.fr

Modèle fictif de véhicule utilisé ; Diesel ; Citadine actuelle ; Tarif du diesel en Italie en novembre 2019 ; Diesel 1,48 €/I d'après comité national de la route (http://www.cnr.fr/Indices-Statistiques/EspaceGazole/Prix-Europe ) 
Le Tableau 1 résume cette ambition. Cependant, il ne prétend pas fournir une comparaison exhaustive et parfaite des coûts-bénéfices de la pratique du terrain à vélo par rapport à ceux de la voiture. L'ambition est surtout descriptive et évocatrice. Néanmoins, pour évaluer plus entièrement les coûts et les bénéfices de cette itinérance, il s'agit également d'intégrer le coût de mes trajets « retours » en train (Tableau 2).

Tableau 2. Descriptif des retours en train et comparaison des "coûts" du voyage par rapport à un voyage fictif en voiture

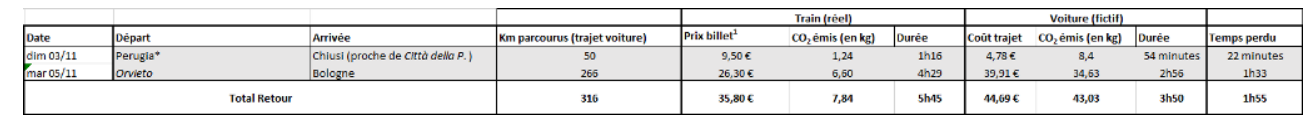

Lecture :

1 : prix du billet incluant $3 € 50$ de frais pour la réservation d'un emplacement vélo.

2 : cout du trajet comprenant le coût en carburant et en péage pour le trajet Orvieto-Bologne.

Calcul de la quantité de CO2 émise lors d'un trajet en train :

La quantité de CO2 émise lors d'un trajet en train est calculée en multipliant la distance parcourue par la quantité moyenne de CO2 émise par un voyageur qui parcoure un kilomètre exprimé en gramme de CO2 /voyageur.km.

Source : basées sur les consommations d'énergie d'un TER français (source : Réseau de transport d'électricités (Rte), 2019) et les données fournies par les institutions françaises sur la fréquentation moyenne des trains. Données indisponibles pour les trains régionaux italiens.

Les facteurs d'émission de l'électricité pour les pays européens (Allemagne, Espagne, UK, Belgique, Pays-Bas) sont issus de la « Base Carbone » de l'ADEME : http://www.bilans ges.ademe.fr/fr/ basecarbone/donneesconsulter/liste-element ?recherche =Electricit \%C3 \%A9+hors+France

26 Au total (Tableau 3) en considérant mon trajet aller (à vélo) et retours (en train), j'ai économisé un peu plus de $55 €$ par rapport à un voyage identique qui aurait été effectué en totalité en voiture. J'ai évité l'émission de plus de $116 \mathrm{~kg}$ de $\mathrm{CO}^{2}$. En contrepartie, par rapport à des déplacements effectués en voiture, j'ai passé quasiment 25 heures de plus à me déplacer ! Les mécanismes de substitution et d'arbitrages entre la pratique du vélo et celle de la voiture sont très clairs. L'économie de carburant (en euros) et d'émission de $\mathrm{CO}^{2}$ permis par la pratique du vélo est contrebalancée par un rallongement conséquent du temps de trajet et la production d'un effort physique important par rapport à celui demandé par la conduite automobile.

Tableau 3. Comparaison des "coûts" du voyage aller-retour en vélo et train par rapport à un voyage fictif en voiture

\begin{tabular}{|c|c|c|c|c|c|c|c|c|}
\hline & & \multicolumn{3}{|c|}{$\begin{array}{l}\text { Vêlo - Train (réel) } \\
\end{array}$} & \multicolumn{3}{|c|}{ Voiture (fictif) } & \multirow[b]{2}{*}{ Temps perdu } \\
\hline & Km parcourus & coutt & $\mathrm{CO}_{2}$ émis (en kg) & Durée & cout trajet & $\mathrm{CO}_{2}$ émis (en kg) & Durée & \\
\hline Total aller & 427 & $0,00 €$ & 0,00 & $33 \mathrm{~h} 55$ & $45,90 €$ & 81,2 & $10 \mathrm{~h} 58$ & $22 \mathrm{~h} 57$ \\
\hline Total retour & 316 & $35,80 €$ & 7,84 & 5 h45 & $44,69 €$ & 43,03 & $3 \mathrm{~h} 50$ & $1 \mathrm{~h} 55$ \\
\hline Total aller-retour & 743 & $35,80 €$ & 7,84 & $39 \mathrm{~h} 40$ & $90,59 €$ & 124,23 & $14 h 48$ & $24 h 52$ \\
\hline
\end{tabular}

Au regard du calcul socioéconomique de l'utilité et de la pertinence de mon choix de mode de transport (Quinet, 2014), mon itinérance à vélo sera considérée comme nonrentable. Concernant les coûts externes de ces deux types de déplacement, notons tout d'abord, qu'à $16 €$ la tonne de carbone en $2018^{7}$, mes $116,4 \mathrm{~kg}$ de $\mathrm{CO}^{2}$ économisés à vélo correspondent à un équivalent monétaire de $1,86 €$ d'économisé pour la planète! Puis, par rapport aux coûts internes, sans rentrer dans le détail de la comparaison monétaire du coût généralisé de mes déplacements à vélo par rapport aux coûts de déplacements effectués en voiture ${ }^{8}$, il est assez aisé de comprendre que le vélo parait moins pertinent que la voiture. Effectivement, selon une approche purement économique, mes déplacements à vélo n'ont fait économiser que très peu d'argent à mon employeur 
$(55 €)$. En contrepartie, par rapport à la durée fictive de déplacements en voiture (14 h 48), mon temps total de déplacement en bicyclette et en train ( $39 \mathrm{~h} \mathrm{40)}$ m'a fait perdre $24 \mathrm{~h} 52$. Sachant que j'ai travaillé sur mon ordinateur, durant près de la moitié de mon temps de trajet en train, on peut considérer que le mode ferré m'a fait économiser $2 \mathrm{~h} 52$ de travail. À partir de ces calculs de coin de table, on note que le vélo m'a fait perdre 22 heures, soit plus de 3 jours de travail à 7 heures quotidiennes; cela correspond à une perte de production de richesse qui, au prorata de mon salaire horaire, s'élève à plus de $300 €$ !

Cependant, dans cette situation, le calcul de l'intérêt d'un mode de transport par rapport à un autre n'est certainement pas si simple que cela, et est donc certainement en partie erroné. En faisant cette itinérance à vélo, je cherche à sensibiliser la communauté scientifique aux enjeux écologiques des déplacements des chercheurs, j'attends, ou a minima espère, par conséquent des bénéfices de plus long terme. D'autre part, en me déplaçant lentement à vélo, je pense avoir appris bien plus que devant un écran d'ordinateur pendant 3 jours. Je suis arrivé à des constats dont je n'aurais pu me rendre compte avec la même certitude, la même efficacité et la même sensibilité en me déplaçant en voiture ou en travaillant uniquement sur la longueur de mon clavier d'ordinateur.

\section{La lenteur, la fatigue et les aléas liés au déplacement : une autre approche face aux exigences de productivité ?}

29 Malgré la variabilité de l'effort fourni selon les jours, je peux néanmoins essayer de décrire certains invariants dans l'organisation de mes journées. Elles sont principalement rythmées par mes coups de pédales, les enquêtes (observations in situ, entretiens, analyses d'archives) dans les Cittàslow et le repos. Grosso modo, l'organisation de mes journées de travail en itinérance résulte de la conciliation entre les contraintes liées à la durée du trajet que je dois réaliser quotidiennement, et la disponibilité des interlocuteurs que je veux interviewer dans lesdites villes. Selon un premier type d'organisation, je quitte la ville de départ le matin après un petit-déjeuner copieux, rejoins la ville d'arrivée en début d'après-midi et réalise dans la foulée mes enquêtes, je retranscris mes analyses et travaille le soir, quitte la ville le lendemain matin. Dans un second cas, je réalise un ou plusieurs entretiens durant la matinée, je voyage ensuite durant l'après-midi, arrive dans la ville d'arrivée le soir, travaille à l'hôtel, réalise de nouveaux entretiens le lendemain matin avant de quitter à nouveau la zone pour me diriger vers une nouvelle ville.

Outre le constat de la diminution de mon empreinte écologique à vélo par rapport à des déplacements qui auraient dû se réaliser en voiture, cette expérience me permet de mieux cerner ce que représentent la notion de décélération des rythmes de vie et les moyens permettant potentiellement d'y accéder. En fait, en me déplaçant à vélo entre différents terrains, je n'ai pas vraiment eu le sentiment de ralentir mon rythme de vie par rapport à une situation fictive avec un autre moyen de transport plus rapide. Au contraire, je n'ai rarement eu le sentiment d'être aussi éprouvé et débordé au travail que lors de ces enquêtes de terrain! Cela s'explique certainement par le fait que j'ai conservé une logique de maximisation de la production, tout en mobilisant volontairement un mode de déplacement lent, c'est-à-dire un outil de production qui était sous-optimal du point de vue de sa rentabilité (unité produite, ou kilomètres 
parcourus par unité de temps) : j'ai probablement commis l'erreur de vouloir faire autant que si j'avais été en voiture, en changeant de ville chaque jour.

31 Se déplacer lentement, être soumis à la fatigue physique, aux aléas climatiques, aux incertitudes quelles qu'elles soient, demande en fait de prendre du recul, certes par rapport à la vitesse de manière générale, mais surtout par rapport aux exigences de productivité dans le cadre de son activité professionnelle.

32 J'ai été rattrapé de temps à autre par la nuit (figure 6) tandis qu'il me restait plusieurs dizaines de kilomètres à parcourir avant de rejoindre mon lieu de résidence. J'ai aussi été quelques fois ralenti, voir stoppé par la pluie (photo 5).

Figure 6. Je n'ai pas de lumières sur mon vélo... (En direction de Pratolino, 25 octobre 2019)

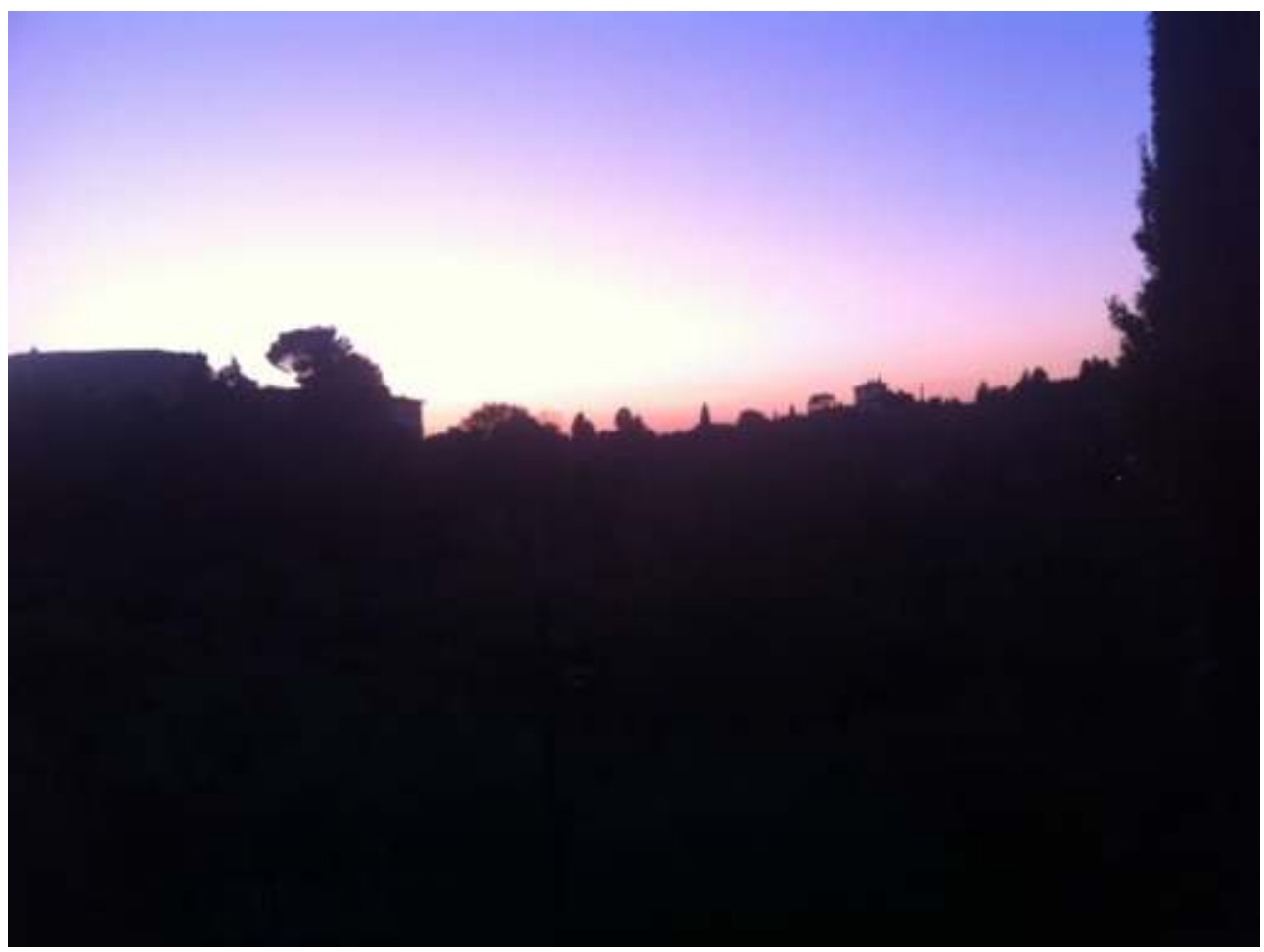

Crédits : Emmanuel Munch. 
Figure 7. 1 h30 sous un tunnel piéton en attendant que l'orage passe (En direction d'Orvieto, 3 novembre 2019)

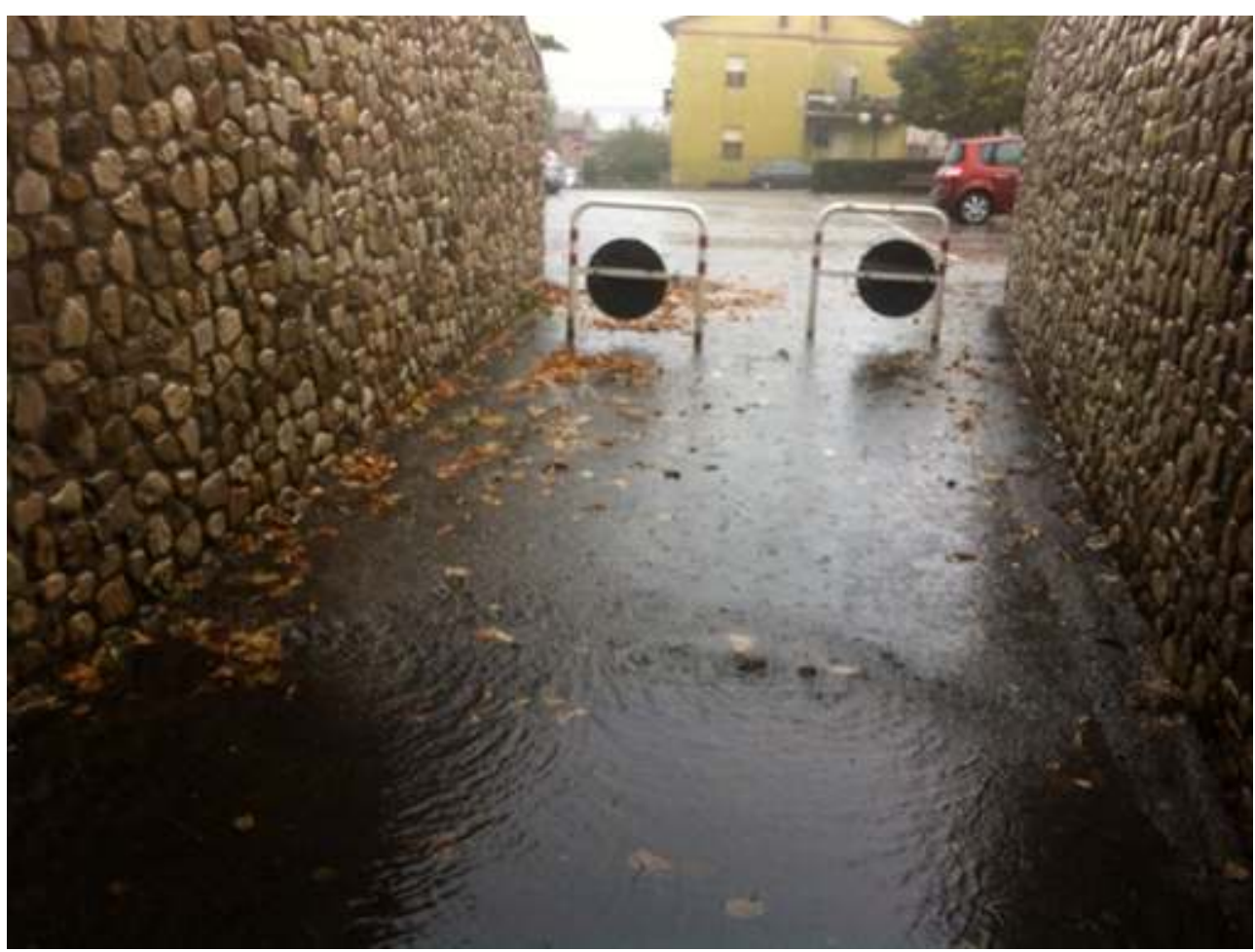

Crédits : Emmanuel Munch.

D'autres fois, j'ai commis des erreurs de calcul en sous-estimant les distances à parcourir sur la carte. Tout ceci aurait pu compliquer grandement mon travail d'enquête puisque malgré les aléas dont je dépendais, je ne pouvais me permettre d'être en retard aux rendez-vous fixés pour mes entretiens dans les différentes villes. Pour faire face à ces difficultés, certaines tactiques peuvent aider. D'une part, je tentais d'organiser les entretiens la veille ou l'avant-veille de mon arrivée prévue sur le site de l'entretien. Cela me permettait d'ajuster mon agenda en fonction de la progression plus ou moins fluctuante de mon avancée à vélo. D'autre part, dans le cas limite où j'avais sous-estimé le temps nécessaire pour rejoindre mon lieu d'entretien, j'ai pu compter sur l'aide de personnes motorisées. Par exemple, le vendredi 25 octobre matin, je me suis fait déposer en véhicule sur le lieu de mon entretien par Vincente et Graziella, deux retraités en vacances dans les Apennins (figure 8). Ils m'ont ensuite gentiment ramené à l'endroit où j'avais laissé mon vélo pour que je puisse reprendre mon itinéraire ${ }^{9}$. 
Figure 8. Vincente e Graziella, grazie ancora ! (à proximité de Marradi, 3 novembre 2019)

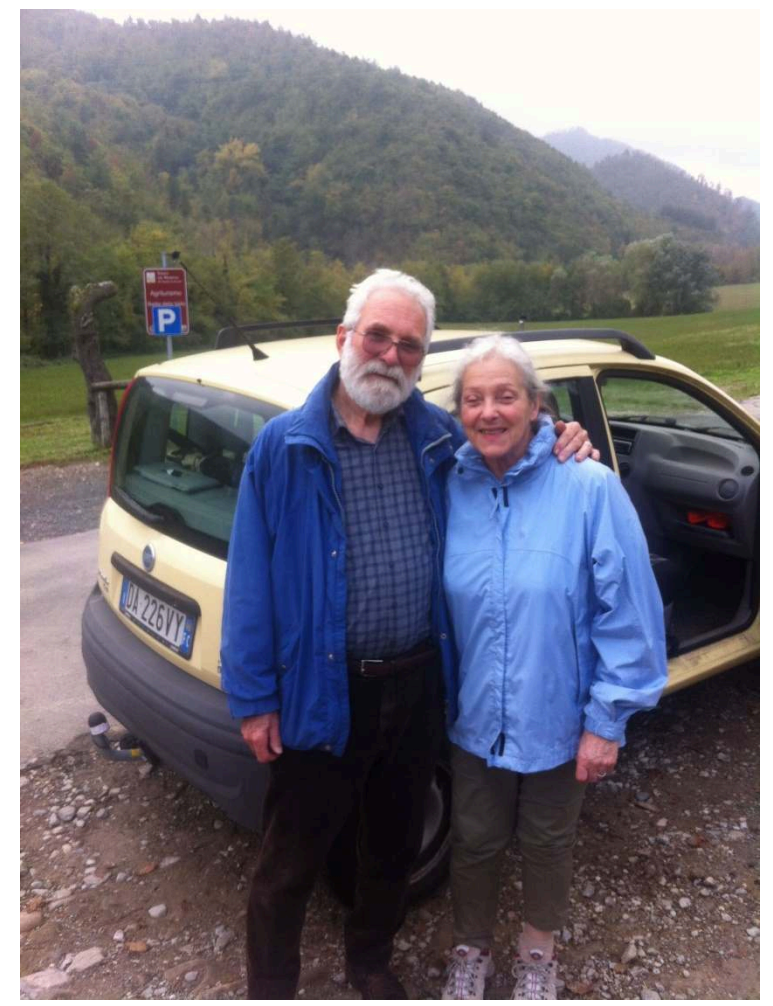

Crédits : Emmanuel Munch.

\section{L'image du cyclotouriste : biais de posture lors de la conduite d'entretien}

Hormis cette fois où je me suis fait déposer en voiture et ai fait une entorse à mon protocole de déplacement, hormis certaines configurations où je pouvais déposer mes affaires à l'hôtel et me doucher avant de rencontrer des élus, la plupart du temps, j'arrivais en tenue de cycliste avec mon enregistreur et mon bloc-notes dans le bureau du maire (figure 9)! Notons que ces difficultés sont surtout liées au manque de temps pour réaliser les enquêtes, plus qu'à l'itinérance à vélo, à proprement parlé. En tant que telle, l'itinérance à vélo n'empêche pas d'arriver la veille sur le lieu d'entretien et de pouvoir ensuite s'y rendre reposé, avec une tenue de rechange (qui certes aura peu de chance d'être un costume repassé). 
Figure 9. Arrivée à vélo au secrétariat général de l'association Cittàslow (Orvieto, 3 novembre 2019)

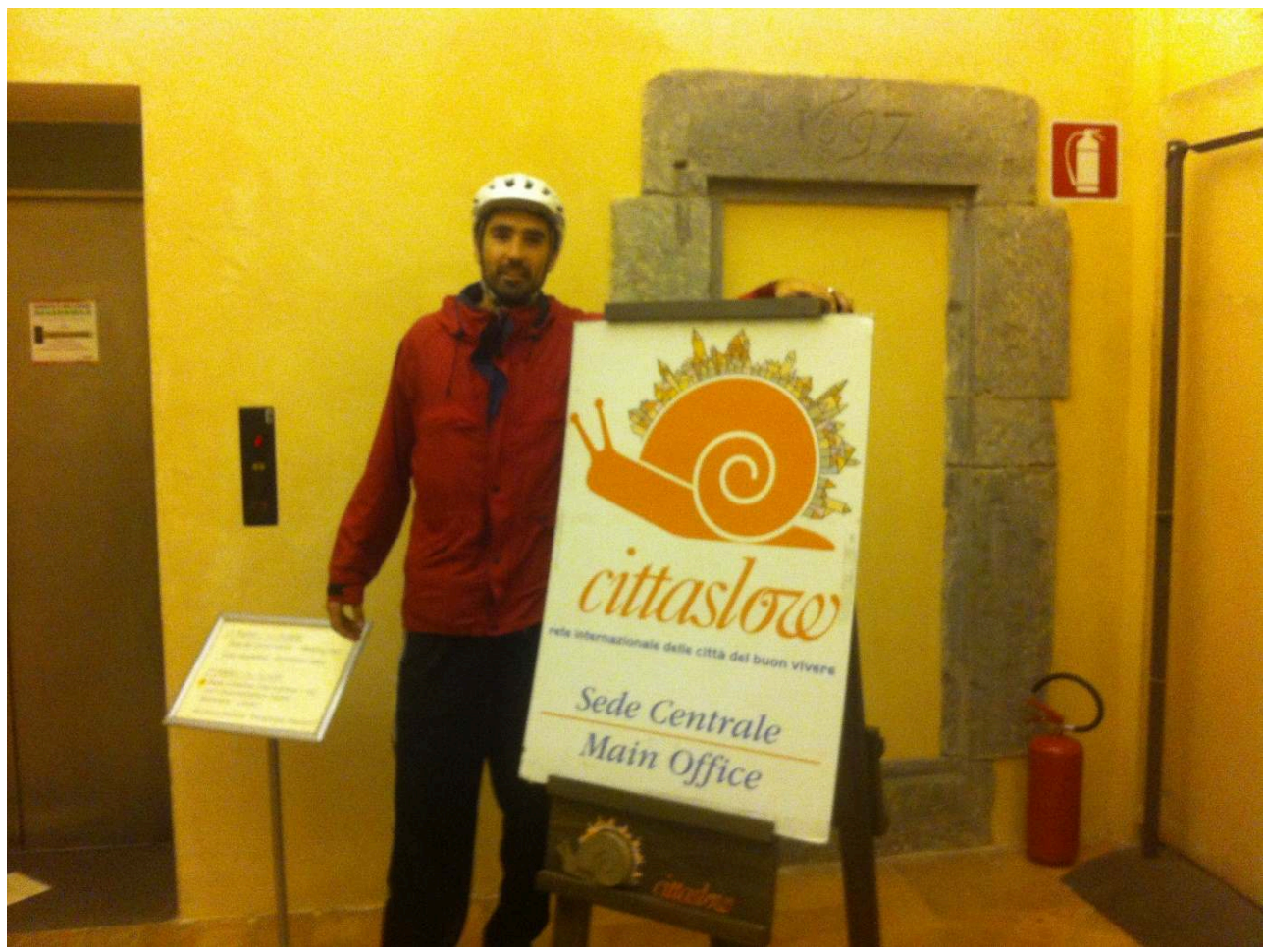

Crédits : Emmanuel Munch.

La tenue dans laquelle je me présente soulève bien évidemment des enjeux sur la posture de l'enquêteur lors de la conduite d'entretien (Demazière 2008). Assurément, en me retrouvant dans le bureau d'élus locaux, ma situation de jeune chercheur me place de facto en position de dominé dans la conduite de l'entretien. La situation professionnelle du maire, son âge, sa maîtrise du langage et des gestes (italien), l'intimidation par l'espace (le bureau avec les dorures, le drapeau italien, la photo du Président italien...) sont autant d'éléments qui laissent penser que je suis en décalage par rapport au milieu de l'élite politique et économique auquel appartiennent mes enquêtés.

L'attitude classique de tout bon enquêteur en sciences sociales consisterait à tenter de niveler ce rapport de force, à tenter de s'élever dans les codes de la hiérarchie sociale en s'habillant par exemple en costume, en faisant subtilement état de son curriculum vitae (Beaud et Weber, 2010 ; Blanchet et Gotman, 2010). Imaginez alors un instant ce qui se peut se produire lorsqu'un jeune chercheur français déguisé en cycliste et parlant un Italien scolaire tente de réaliser un entretien avec un élu italien. À cet égard, je dois avouer que j'ai été surpris que l'on m'accorde quasi systématiquement l'accès au bureau municipal. Ceci est peut-être lié au caractère rural et extrêmement accueillant des villes enquêtées. Cela doit également avoir un lien avec le potentiel intérêt promotionnel pressenti par les enquêtés: s'ils acceptaient d'être interviewés, ils auraient de bonnes chances de voir leur ville paraître en bonne position dans les publications qui pourraient en découler.

37 Cela a néanmoins produit quelques saynètes cocasses. À la magnifique mairie de Città della Pieve, avant de m'accorder un entretien, on a longuement insisté pour que je fournisse un certificat de l'université attestant de ma qualité de chercheur en cuissard ! 
Si votre apparence ne peut justifier de vos qualités, lorsque vous êtes un chercheur cyclotouriste, prévoyez tout de même vos papiers, pour éviter d'être taxé de vagabondage!

\section{Le vélo : un moyen d'accès résonnant avec le territoire}

38 Même s'il complique certainement l'accès aux entretiens avec des "personnalités ", du point de vue de l'accès aux matériaux d'observation, le vélo permet de saisir des moments au vol, des photographies, des scènes figées dans la fluidité du voyage sans avoir besoin de s'arrêter (figure 10).

Figure 10. Temps suspendu (Castelnuovo Berardenga, 28 octobre 2019)

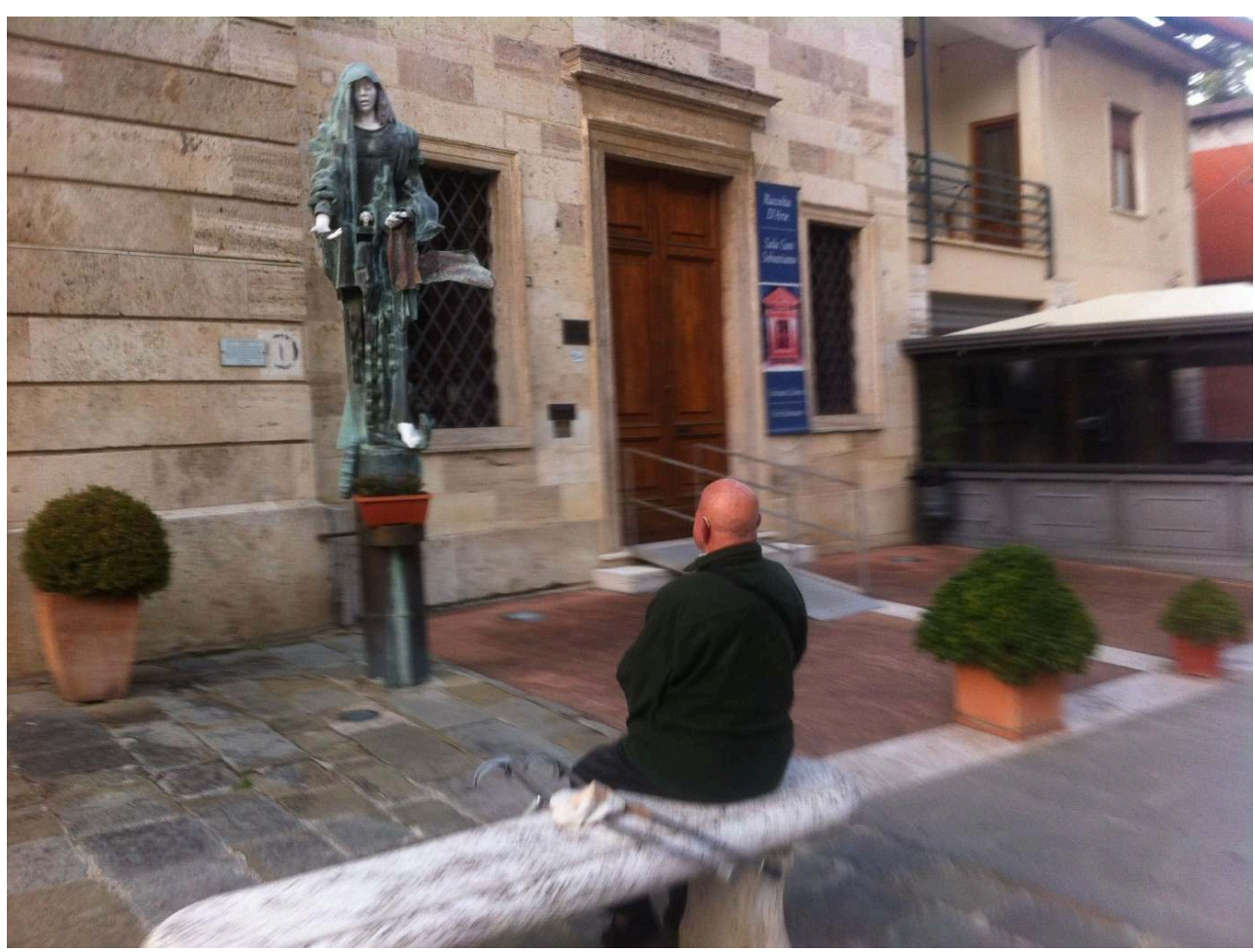

Crédits : Emmanuel Munch.

Plus globalement, par rapport à la voiture, le vélo permet de « ressentir » ses terrains d'enquête, ou encore selon Hartmut Rosa, de "résonner» (2018) avec son environnement. En géographie, Wunderlich (2008) parle de la marche en tant que mode de déplacement faisant vibrer tous les sens, et en particulier la vue, le toucher. Comme si ces modes lents, en s'insérant dans des échelles spatio-temporelles plus fines, donnaient effectivement la sensation qu'on pouvait toucher avec plus d'acuité le territoire traversé (figure 11). Ce serait donc bien parce que la lenteur mobilise tous nos sens qu'elle nous oblige à prendre conscience de ce qui nous entoure. 
Figure 11. Contemplation (en direction de Florence, 26 octobre 2019)

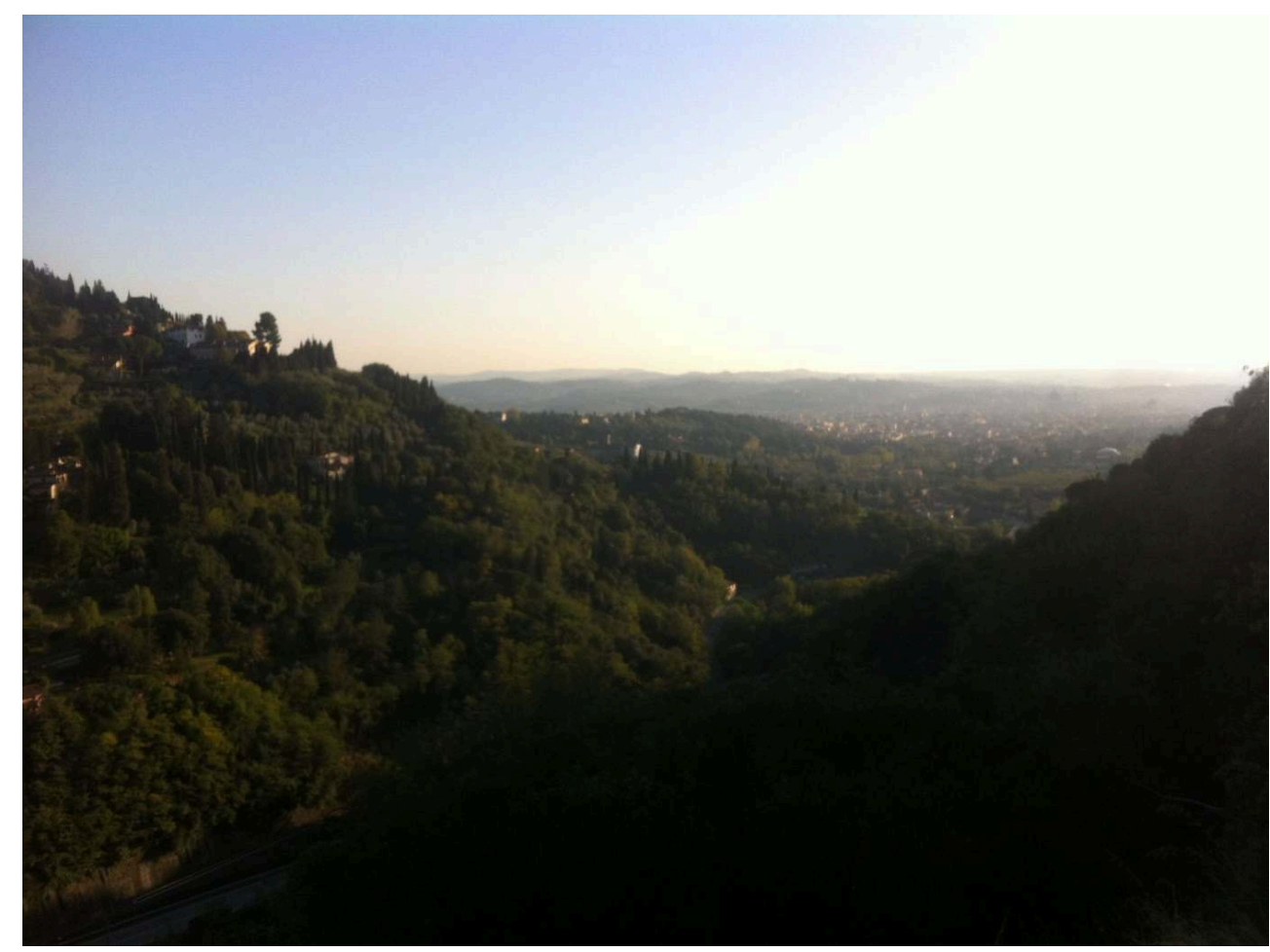

Crédits : Emmanuel Munch.

40 En cheminant lentement, on perçoit de façon plus marquée l'histoire de l'action anthropique sur les territoires enquêtes, l'intensité du dynamisme économique et démographique, les particularismes locaux (Blanchard, 1963 ; de Certeau, 1990). Avant d'arriver dans une ville enquêtée, et au-delà de tous les diagnostics statistiques envisageables, il n'existe certainement pas de meilleure exploration territoriale que celle fournie par la lenteur du déplacement, l'épreuve de la contemplation à pied ou à vélo (Bonnamour, 2000). Passer à côté d'une centrale électrique désaffectée (figure 12), à la vitesse de $20 \mathrm{~km} / \mathrm{h}$ et à l'air libre, produit une sensation autrement plus marquée que lorsqu'on la longe brièvement dans l'habitacle de sa voiture. Descendre d'infinis champs d'oliviers (figure 13) à vélo donne parfois l'impression de devenir soi-même une délicieuse olive roulante, ou a contrario confronté à l'ascension, un vulgaire oléagineux que l'on triture! 
Figure 12. Traversée pesante (en direction de Pérouse, 30 octobre 2019)

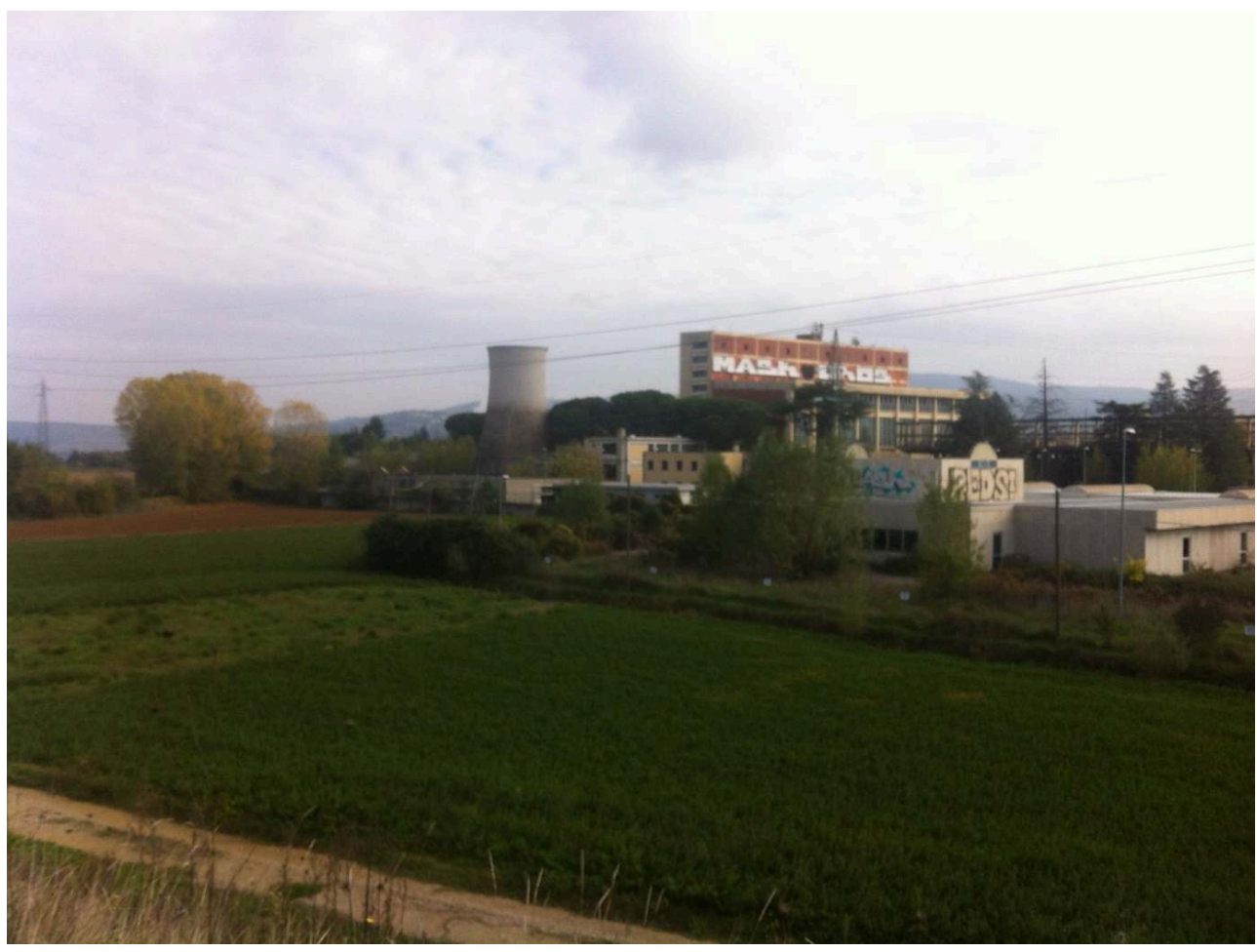

Crédits : Emmanuel Munch.

Figure 13. Traversée légère (en direction de Castelnuovo Berardenga, 28 octobre 2019)

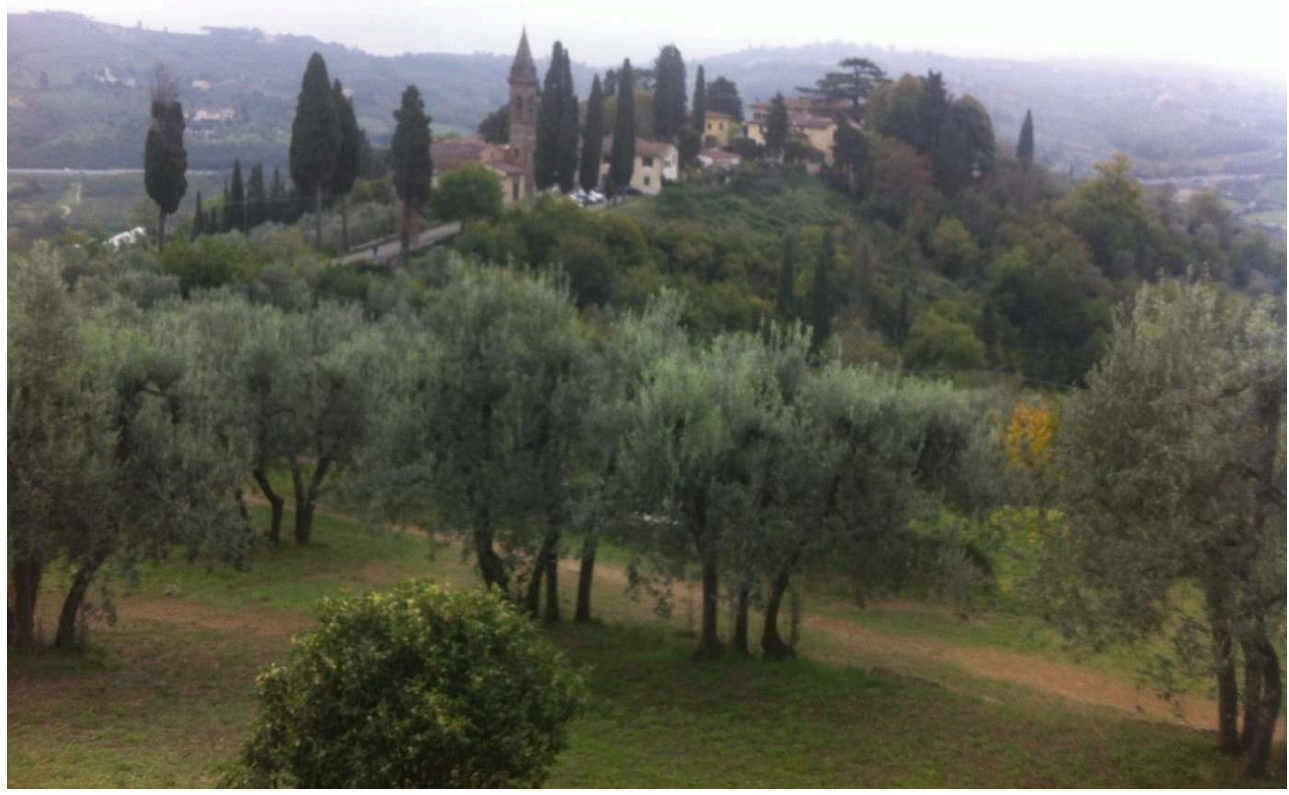

Crédits : Emmanuel Munch. 
41 Rendu accessible par la lenteur, ce «corps-à-corps avec le réel » aide à ressentir « une unification entre soi et le monde" (Descola, 2005) et en ce sens, participe certainement d'une prise de conscience écologique. Toutefois, dans ce corps à corps, je n'ai pas trouvé la force créatrice et inspiratrice que l'on prête souvent à la ballade. En effet, chercheurs ou écrivains (Solnit, 2004) décrivent fréquemment les vertus de la promenade pour faire émerger de nouvelles idées. Initialement, j'avais moi aussi pensé que mes déplacements à vélo seraient propices à la réflexion. J'ai vite été douché ! L'effort physique ne laissait que très peu de place à l'effort intellectuel. La plupart du temps, j'étais happé par l'instant. Et je reconnais très volontiers que lorsque l'inclinaison du sol offrait un peu plus d'énergie à l'esprit, je me laissais plus facilement emporté par des réflexions qui ne concernaient pas le travail.

En parallèle des intérêts heuristiques, méthodologiques et écologiques des déplacements de terrain effectués à vélo, se cache aussi (pour moi en tout cas), la notion de plaisir et de passion. En effet, j'éprouve du plaisir à me promener sur un vélo et ai fréquemment l'habitude de pratiquer le cyclotourisme pendant mon temps libre. Par ailleurs, pour différentes raisons personnelles, je crois que la problématique de l'organisation des rythmes de vie et de la vitesse et un sujet de réflexion qui titille depuis longtemps ma curiosité.

Dans la cuisine ou dans l'atelier de fabrication des sujets de recherche, bien que cela ne soit que très rarement reconnu, l'attrait pour une problématique ou une passion personnelle guide autant la définition d'un sujet de recherche que l'intérêt scientifique ou l'intérêt social environnant le sujet. Chaque chercheur passionné sait également que, dans la conduite et la pratique de la recherche, ce rapport entre « l'investissement personnel » et la profession scientifique persiste. On dit souvent des chercheurs qu'ils sont habités personnellement par leur questionnement scientifique ou réciproquement, c'est selon, scientifiquement habités par leurs passions personnelles.

Seulement, ce fait est très souvent occulté (Calberac, 2010 ; Volvey, 2004) au prétexte d'objectifs de distanciation, ou de neutralisation axiologique et affectif du rapport entretenu entre le chercheur et son objet, ses questions. Ici, j'assume prendre le contrepied de cette posture. En dernier lieu, avec certaines précautions en tête, ce retour réflexif sur ces deux semaines d'itinérances cyclistes est aussi pour moi l'occasion de défendre un point de vue sur les modes de faire de la recherche : lors de la réalisation d'enquêtes de terrain, il peut être fertile de se placer comme un élément faisant - avec ses «vibrations", ses affects - partie intégrante du champ d'investigation épistémologique et méthodologique.

\section{Conclusion}

En prenant comme point d'appui l'histoire d'un chercheur ayant fait le choix de " perdre du temps » à vélo pour se déplacer entre ces différents d'enquête, l'ambition était de montrer que cette démarche pouvait avoir un double intérêt scientifique.

Premièrement, en rendant compte de mon itinérance à vélo entre mes différents terrains d'enquête, j'alimente des débats et des questions relatives à la pratique de la recherche. Je pense que cela nourrit des discussions (Vivet et al., 2015) qui touchent aussi bien à la notion de Slow Science (renommée " désexcellence scientifique ») que la question de l'empreinte écologique générée par les chercheurs en déplacement. 
Témoigner des façons dont il est possible aujourd'hui de ralentir sa pratique de la recherche tout en diminuant son empreinte écologique a, à mon avis, toute sa place, dans la littérature scientifique en Géographie.

Ce carnet de terrain invite à prendre conscience des mérites et des intérêts de la lenteur, en tant que modalités de déplacements écologiques des chercheurs en action. Il invite aussi à prendre conscience des intérêts du vélo pour accéder, observer, toucher, sentir et finalement « résonner » (Rosa, 2018) plus aisément avec son terrain. Toutefois, l'itinérance à vélo entre différents terrains d'enquête s'est accompagnée de nombreuses complications et difficultés à avoir en tête. Il serait malhonnête de dresser un constat trop idyllique de cette expérience. Mais attention au travers de ces remarques, il ne s'agit pas non plus de condamner l'efficacité du déplacement à vélo dans l'absolu. Mon expérience est loin d'être totalement généralisable. Une bonne partie des complications auxquelles j'ai dû faire face sont liées au contexte géographique (dénivelés, absence d'aménagement cyclable). Un compte-rendu de " cyclo-recherche " aux Pays-Bas en été, ne conduirait certainement pas aux mêmes constats et mises en garde.

Au travers de cette expérience, on peut dire que se rendre à vélo sur ses terrains complique le travail du chercheur. Les déplacements cyclistes sont plus dangereux que les déplacements en voiture. Ils peuvent générer une importante fatigue. Dans mon cas, sans la voiture, il est clair que j'arrivais parfois épuisé à mes rendez-vous d'entretien. Aussi, les déplacements peuvent être moins fiables, car soumis aux aléas climatiques. En fait, ces déplacements professionnels, parce qu'ils sont globalement moins performants, moins rapides, invite à reconsidérer ou a minima à être en capacité de prendre du recul par rapport aux exigences de productivité de la recherche.

Deuxièmement et en conséquence, cette initiative autorise un cadrage plus fin des questions posées dans le projet Politiques de Mobilité Durable et Décélération ( $\mathrm{PMD}^{2}$ ) que je poursuis actuellement. En plus de la réduction de mon incidence écologique à vélo par rapport à des enquêtes qui auraient dû se réaliser en voiture, ces deux semaines d'itinérances me fournissent des débuts de réponses sur les moyens permettant de décélérer son rythme de vie (Kirschner, 2020). Alors que c'était une des hypothèses du projet de recherche, j'ai constaté qu'en me déplaçant à vélo entre mes différents terrains, je n'ai pas vraiment eu le sentiment de ralentir mon rythme de vie ou de travail par rapport à une situation fictive avec un autre moyen de transport plus rapide.

A contrario, je ne me suis jamais senti aussi éprouvé et débordé au travail que lors de ces enquêtes de terrain. D'autres chercheurs ayant eux-aussi effectué des enquêtes à vélo avait déjà indiqué à quel point, il pouvait être physiquement et mentalement éprouvant de combiner la dépense physique de la pratique cycliste avec la dépense mentale de la pratique de l'entretien (Palmer, 1996; Spinney, 2006). Dans mon cas, ce sentiment inattendu d'accélération du rythme de travail a certainement été accentué par le fait que j'ai voulu faire autant d'enquêtes que si j'avais été en voiture, en changeant de villes chaque jour. Moralité : pour décélérer, agir seulement sur les déplacements ne suffit pas.

51 Se déplacer plus lentement et de façon non-polluante ne semble pas être une entrée efficace pour imposer un rythme de production plus ralenti et plus favorable à la création de connaissances solides et approfondies. Les choses doivent plus nettement 
s'opérer en sens inverse : il est probable que ce soit surtout un rythme de production apaisé qui autorise la lenteur des déplacements.

En tout état de cause, sans pouvoir isoler avec certitude le sens des causalités, il est clair que si on se déplace lentement dans le cadre de son activité professionnelle, il faut pouvoir choisir de moins travailler, ou de passer plus de temps sur chacune des tâches afin de ressentir « les bienfaits de la décélération » (Munch et Zachariou, 2020). Si l'on reformule cette observation personnelle sous forme d'hypothèse de recherche, on peut envisager que le recours à la dénomination slow traduit, plus qu'une aspiration au ralentissement, la volonté de prendre le pouvoir sur les rythmes économiques, individuels et collectifs et de choisir ainsi les modalités de vécu du temps quotidien (Diestchy, 2015). Théoriquement, cela signifie que le contrepoint du sentiment "d'accélération des rythmes de vie " ne serait pas tant la décélération, mais le loisir compris comme un libre usage du temps dans son contenu et dans son rythme (Godillon et al., 2015). Autant d'hypothèses qui, appliquées dans un premier temps à mon cas, ont facilité par la suite une exposition plus claire et plus explicite des interrogations aux enquêtés rencontrés dans les différentes Cittàslow italiennes.

Enfin, au-delà des perspectives que cette expérience ouvre pour ma propre recherche, j'aimerais qu'elle puisse annoncer des lendemains plus heureux pour la communauté scientifique. Aujourd'hui, je pense qu'il est important d'exercer une forme de résistance (de Coninck, 1998) pour permettre à d'autres de prendre eux aussi le temps dans la lenteur (Munch, 2019). Durant ce terrain à l'étranger, j'ai eu la chance incroyable de travailler sans pression, avec confiance, et avec des moyens financiers plus que satisfaisants. Ces conditions, de plus en plus rares, ont rendu possible une pratique de la recherche réflexive et ralentie. Le temps laissé au temps ne va plus de soi. Il faut donc travailler à rendre légitime, à donner de la visibilité et à montrer la faisabilité et l'intérêt de la lenteur dans la recherche! Elle fait partie du métier de chercheur. Les processus extrêmes d'accélération sociale et culturelle sont au cœur de l'ère de l'information. Pourtant la recherche, pour l'essentiel, continue d'être une affaire lente et patiente. Dans ce contexte, il faut a minima accepter de s'interroger sur la manière dont la recherche doit répondre à l'accélération de la vie sociale et de la culture. Doitelle tenter de suivre le rythme d'un monde qui change plus vite que jamais ? Ou la force de la recherche réside-t-elle dans le fait qu'elle est une forme lente, détachée et réfléchie qui se situe en dehors de la logique accélérée et polluante de la culture capitaliste contemporaine?

BIBLIOGRAPHIE

ADMINAITE-FODOR D., JOST G. (2020), « How safe is walking and cycling in Europe? », PIN Flash Report, no 38. Brussels: European Transport Safety Council. [En ligne : https://trid.trb.org/View/ 1696180].

BAUDELLE G., OZOUF-MARIGNIER M.-V., ROBIC M.-C. (2001), Géographes en pratiques (1870-1945). Le terrain, le livre, la cité, Rennes, Presses Universitaires de Bretagne. 
BEAUD S., WEBER F. (2010), Guide de l'enquête de terrain, Paris, La Découverte.

BLANCHET, A., GOTMAN A. (2010), L'entretien. L'enquête et ses méthodes, Paris, Armand Colin.

BIGO A. (2020), « Vitesse des mobilités : accélération au 20ème siècle, ralentissement au 21e ? ", Publications de la Chaire Energy-Prosperity. [En ligne : http://www.chair-energy prosperity.org/ publications/vitesse- mobilites-france/]

ADAM B. (2004), Time, Cambridge, Polity Press.

ANDERSON L. (2006). « Analytic Autoethnography », Journal of Contemporary Ethnography, vol. 35, no. 4, pp. 373-95. [En ligne : https://doi.org/10.1177/0891241605280449]

BISSELL D. (2010), « Narrating Mobile Methodologies : Active and Passive Empiricisms ", in Fincham B., McGuinness M., et Murray L., Mobile Methodologies, London, Palgrave Macmillan UK, pp. 53-68.

BLANCHARD R. (1963), Je découvre l'université. Douai, Lille, Grenoble, Paris, Fayard.

BROWN K., SPINNEY J. (2010), « Catching a Glimpse : The Value of Video in Evoking Understanding and Representing the Practice of Cycling ", in Fincham B., McGuinness M., Murray L., Mobile Methodologies, London, Palgrave Macmillan UK, pp. 130-51.

BONNAMOUR J. (2000), Du bonheur d'être géographe. Fontenay-aux-Roses, ENS Editions.

BÜSCHER M., URRY J., WITCHGER K. (2010), Mobile Methods, London, Routledge.

BUTTIMER A. (1983), The Practice of Geography, Harlow, Longma.

CALBERAC Y. (2010), « Terrains de géographes, géographes de terrain. Communauté et imaginaire disciplinaires au miroir des pratiques de terrain des géographes français du XXe siècle ", Thèse de doctorat en géographie, Université Lyon II.

de CERTEAU M. (1990) [1980], L'Invention du quotidien, 1. : Arts de faire , Paris, Gallimard.

CLAVAL P. (2013), « Le rôle du terrain en géographie, Des épistémologies de la curiosité à celles du désir ", Confins, Revue franco-brésilienne de géographie / Revista franco-brasilera de geografia, no. 17. [En ligne : https://doi.org/10.4000/confins.8373]

COLLIGNON B. (2010), «L'éthique et le terrain », L'Information geographique, vol 74, no. 1, pp. 63-83. [En ligne : https://doi.org/10.3917/lig.741.0063]

de CONINCK, F. (1998), « Point de vue : les bonnes raisons de résister au changement », Revue française de gestion, vol. 120, pp. 162-168.

DEMAZIERE D. (2008), « L'entretien biographique comme interaction négociations, contreinterprétations, ajustements de sens ». Langage et société, vol. 123, no. 1, pp. 15-35. [En ligne : https://doi.org/10.3917/ls.123.0015]

DESCARREGA B., MOATI P. (2016), « Modes de vie et mobilité. Une approche par les aspirations. Phase quantitative », Rapport de recherche du Forum Vies Mobiles, ObsoCo. [En ligne : http:// owncloud.forumviesmobiles.org/index.php/s/weZAhOGZm8OtAJj].

DESCOLA P. (2005), Par-delà nature et culture, Paris, Gallimard.

DIESTCHY M. (2015), « Tensions et compromis dans les valeurs spatiales du slow », Carnets de géographes, no. 8. [En ligne : https://doi.org/10.4000/cdg.291]

EDENSOR T. (2000), «Walking in the British Countryside: Reflexivity Embodied Practices and Ways to Escape », Body \& Society 6, no. 3-4, pp. 81-106. [En ligne : https://doi.org/ $10.1177 / 1357034$ X00006003005] 
FINCHAM B. (2006), « Back to the 'Old School': Bicycle Messengers Employment and Ethnography », Qualitative Research, vol. 6, no. 2, pp. 187-205. [En ligne : https://doi.org/ 10.1177/1468794106062709]

GANE N. (2006), « Speed up or slow down? Social theory in the information age », Information Communication \& Society, vol. 9, no. 1, pp. 20-38. [En ligne : https://doi.org/ $10.1080 / 13691180500519282]$

GODILLON S., LESTEVEN G., MALLET S. (2015), « Réflexions autour de la lenteur », Carnets de géographes, no 8. [En ligne : https://doi.org/10.4000/cdg.281]

ILLICH I., [1975], 2004, Énergie et Équité, dans Euvres complètes, Volume 1, Paris, Fayard, pp. 383-447.

KIRSCHNER C. (2020), « Le rythme singulier et créatif de l'itinérance », EspacesTemps,net Revue électronique des sciences humaines et sociales, avril. [En ligne : https://doi.org/10.26151/ espacestemps.net-eymb-y328]

LARSEN J. (2014), « (Auto)Ethnography and cycling », International Journal of Social Research Methodology, vol. 17, no. 1, pp. 59-71. [En ligne : https://doi.org/10.1080/13645579.2014.854015].

LES ÊTRES LOIRE. (2020), » Paysage à la dérive », Openfield, no 15. [En ligne : https://www.revueopenfield.net/2020/07/01/paysage-a-la-derive/]

MUNCH E. (2019), « Slow life, on vit trop vite, comment ralentir? », Rencontres du CNRS Sciences et Citoyens, Caen, 23 mars 2019. [En ligne : https://normandie.cnrs.fr/les-rencontres-du-cnrssciences-et-citoyens/]

MUNCH E., BELTON CHEVALLIER L., SIMON G. (2020). Rapport intermédiaire du projet PMD², ADEME. MUNCH E., ZACHARIOU L. (2020), « Ralentir pour gagner du temps », Urbanisme, Dossier spécial : les nouveaux paradigmes de la mobilité, no. 419, pp. 39-41.

NEVINS J. (2014), « Academic Jet-Setting in a Time of Climate Destabilization: Ecological Privilege and Professional Geographic Travel », The Professional Geographer, vol. 66, no. 2, pp. 298-310.

PALMER C. (1996). « A Life of Its Own : The Social Construction of the Tour de France », Doctoral Thesis.

QUINET E. (2014), « L'évaluation Socioéconomique des Investissements Publics », PSE Working Papers, HAL.

ROSA H. (2018), Résonance : Une sociologie de la relation au monde, Paris, Éditions La Découverte, $536 \mathrm{p}$.

SPINNEY J. (2006), « A Place of Sense: A Kinaesthetic Ethnography of Cyclists on Mont Ventoux », Environment and Planning D: Society and Space, vol 24, no 5, pp. 709-32.

SOLNIT R. (2004), L'art de marcher. (trad.de l'Anglais), Arles/Montréal, Actes Sud.

STENGERS I. (2016), Une autre science est possible ! Manifeste pour un ralentissement des sciences, Paris, La Découverte.

VIVET J., COLLIGNON B., CHIVALLON C. (2015), « Entretien croisé avec Béatrice Collignon et Christine Chivallon sur la lenteur dans la science et la pratique actuelle des géographes ", Carnets de géographes, no. 8. [En ligne : http://journals.openedition.org/cdg/285]

VOLVEY, ANNE. 2004. “ „Übergänglichkeit“ : ein neuer Ansatz für die Epistemologie der Geographie ». Geographische Zeitschrift, vol 92, no. 3, pp. 170-84.

WIEL M. (2007), Pour planifier les villes autrement, Paris, L'Harmattan. 
WUNDERLICH F. M. (2008), « Walking and Rhythmicity: Sensing Urban Space », Journal of Urban

Design, vol. 13, no. 1, pp. 125-39.

\section{NOTES}

1. L'auteur tient à remercier Gabriele Manella pour son accueil au département de Sociologie de l'Université de Bologne durant l'année 2019-2020, ainsi que son financeur, l'Agence de la Transition Ecologique (Ex-Ademe). Il remercie également ses collègues d'avoir cru en l'intérêt de cette démarche et de lui avoir accordé leur confiance.

2. http://www.cittaslow.org.

3. Recherche en mouvement

4. http://nicolasplain.fr/explore/

5. Les raisons ayant présidé au choix des 6 des villes enquêtées sont détaillées dans le rapport de mi-parcours de la recherche (Munch et al., 2020). En substance, le choix des terrains a été guidé par les objectifs de recherche et les contraintes inhérentes à la conduite de cette dernière (budget, calendrier...). Le voyage à vélo entre ces 6 villes n'était pas prévu avant que la recherche ne débute. Ce n'est qu'au début des investigations que la disposition spatiale des villes intéressantes est apparu comme étant potentiellement compatible avec la pratique cycliste.

6. La proportion de décès de cyclistes due à une collision avec un véhicule motorisé est plus élevée en Italie (83\%) que pour la moyenne de l'UE (77 \%) (Adminaité-Fodor et Jost, 2020).

7. D'après, Ministère de la Transition Écologique et Solidaire (2018) Prix du carbone [archive], publié le 17 déc. 2018, consulté le 11 septembre 2019

8. On considère ici que je possède soit un vélo soit une voiture et que, dans les deux cas, mon employeur n'a donc pas à prendre en compte le coût de la location d'un véhicule.

9. En mentionnant cette rencontre, on pourrait s'attendre à un développement sur le potentiel de sérendipité du déplacement à vélo. Mais dans les faits, les rencontres qui ont permis de nourrir le travail de recherche s'opéraient plutôt à l'intérieur des foyers, lorsque je logeais chez l'habitant.

\section{RÉSUMÉS}

L'article rend compte d'une réflexion consistant à penser d'un même mouvement l'objet et la méthode d'enquête : la décélération. L'auteur présente ainsi une démarche méthodologique et éthique qui permet d'étudier le réseau des Cittàslow italiennes tout en analysant la lenteur du processus de recherche: les déplacements de terrain sont faits à bicyclette. Par ce retour d'expérience, il met en lumière des enjeux peu discutés dans la littérature scientifique, car souvent cantonnés à la «cuisine de la recherche » : l'organisation pratique et écologique d'une enquête, les liens entre création de connaissance, rythme de travail et de déplacement, ou encore le plaisir et la passion qui lient le chercheur à son objet d'étude.

The article reports on a reflection consisting in thinking of the object and the method of investigation in the same movement: deceleration. The author thus presents a methodological and ethical approach that makes it possible to study the network of Italian Cittàslow while analyzing the slowness of the research process: the field research travels are made by bicycle. 
Through this experience feedback, he highlights issues that are rarely present in the scientific literature, as they are often relegated to the "kitchen of research": the practical and ecological organization of a survey, the links between knowledge creation, work and travel rhythm, and the pleasure and passion that link the researcher to his object of study.

\section{INDEX}

Thèmes : Carnets de terrain

Mots-clés : écologie, mouvements Slow, déplacements de terrain, vélo, Italie

Keywords : Ecology, Slow movements, field research travel, cycling, Italy

\section{AUTEUR}

\section{EMMANUEL MUNCH}

Chargé de recherche au Laboratoire Dynamiques, Economiques et Sociales des Transports (DEST), Enseignant à l'Ecole d'Urbanisme de Paris (EUP).

emmanuel.munch[at]enpc.fr 